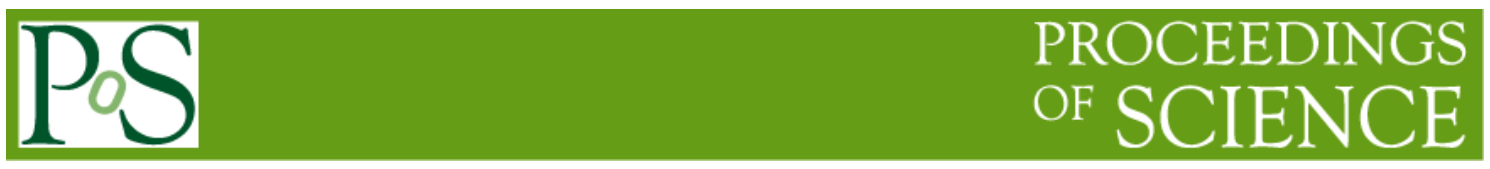

\title{
STAR MAPS Vertex Detector operational experience
}

\section{Leo Greiner $^{1}$ for the STAR collaboration}

Lawrence Berkeley National Lboratory

1 Cyclotron Road MS70R0319

Berkeley CA, USA

E-mail: lcgreiner@1bl.gov

\section{Abstract}

The Heavy Flavor Tracker (HFT) is a set of silicon tracking detectors, which are used by the Solenoidal Tracker at RHIC (STAR) to extend its measurement capabilities in the heavy flavor domain. STAR takes data at the Relativistic Heavy Ion Collider (RHIC) at Brookhaven National Laboratory. This system took data in Au+Au collisions, $\mathrm{p}+\mathrm{p}, \mathrm{p}+\mathrm{Au}$ and $\mathrm{d}+\mathrm{Au}$ collisions at $\sqrt{S_{N N}}=$ $200 \mathrm{GeV}$ at RHIC, during the period 2014 - 2016. The innermost high resolution PiXeL detector (PXL) is the first application of the state-of-the-art thin Monolithic Active Pixel Sensors (MAPS) technology in a collider environment.

The PXL detector is based on $50 \mu \mathrm{m}$-thin MAPS sensors with a pitch of $20.7 \mu \mathrm{m}$. Each sensor includes an array of nearly 1 million pixels, read out in a column parallel rolling shutter mode with an integration time $185.6 \mu \mathrm{s}$. The $170 \mathrm{~mW} / \mathrm{cm}^{2}$ power dissipation allows for air cooling and contributes to reduce the global material budget to $0.4 \%$ radiation length on the innermost layer. The experience and lessons learned from construction and operations of this novel detector are presented. Detector performance and results from $2014 \mathrm{Au}+\mathrm{Au}$ data analysis, demonstrating the STAR capabilities of charm reconstruction, are described.

The 25th International workshop on vertex detectors

September 26-30, 2016

Location

La Biodola, Isola d'Elba, ITALY

\footnotetext{
${ }^{1}$ Speaker

(C) Copyright owned by the author(s) under the terms of the Creative Commons 


\section{STAR Heavy Flavor Tracker Upgrade}

The STAR HFT upgrade was designed to provide the STAR experiment with the capability to perform direct topological reconstruction of heavy flavor decays such as $\mathrm{D}^{0} \rightarrow \mathrm{K} \pi$ with short $\mathrm{c} \tau$ decay lengths of $\sim 100 \mu \mathrm{m}$ [1][2]. This requires a tracking system with excellent Distance of Closest Approach (DCA) pointing resolution. The physics driven requirement for the detector set was a DCA of $60 \mu \mathrm{m}$ for $750 \mathrm{MeV} / \mathrm{c}$ kaons. In order to achieve this, the HFT upgrade was designed as a set of three separate silicon based tracking detectors to be inserted into the inner field cage of the STAR Time Projection Chamber (TPC). The Silicon Strip Detector (SSD) is the outermost layer and is located at a radius of $22 \mathrm{~cm}$ from the beam-line. The Intermediate Silicon Tracker (IST) is a silicon pad detector at $14 \mathrm{~cm}$ radius. The PXL detector consists of two layers of MAPS at a radial distance of 8 and $2.8 \mathrm{~cm}$ respectively and provides the high resolution points leading to the high overall system pointing resolution. All of these detectors are inserted into the TPC inner field cage on cylindrical carbon composite structures.

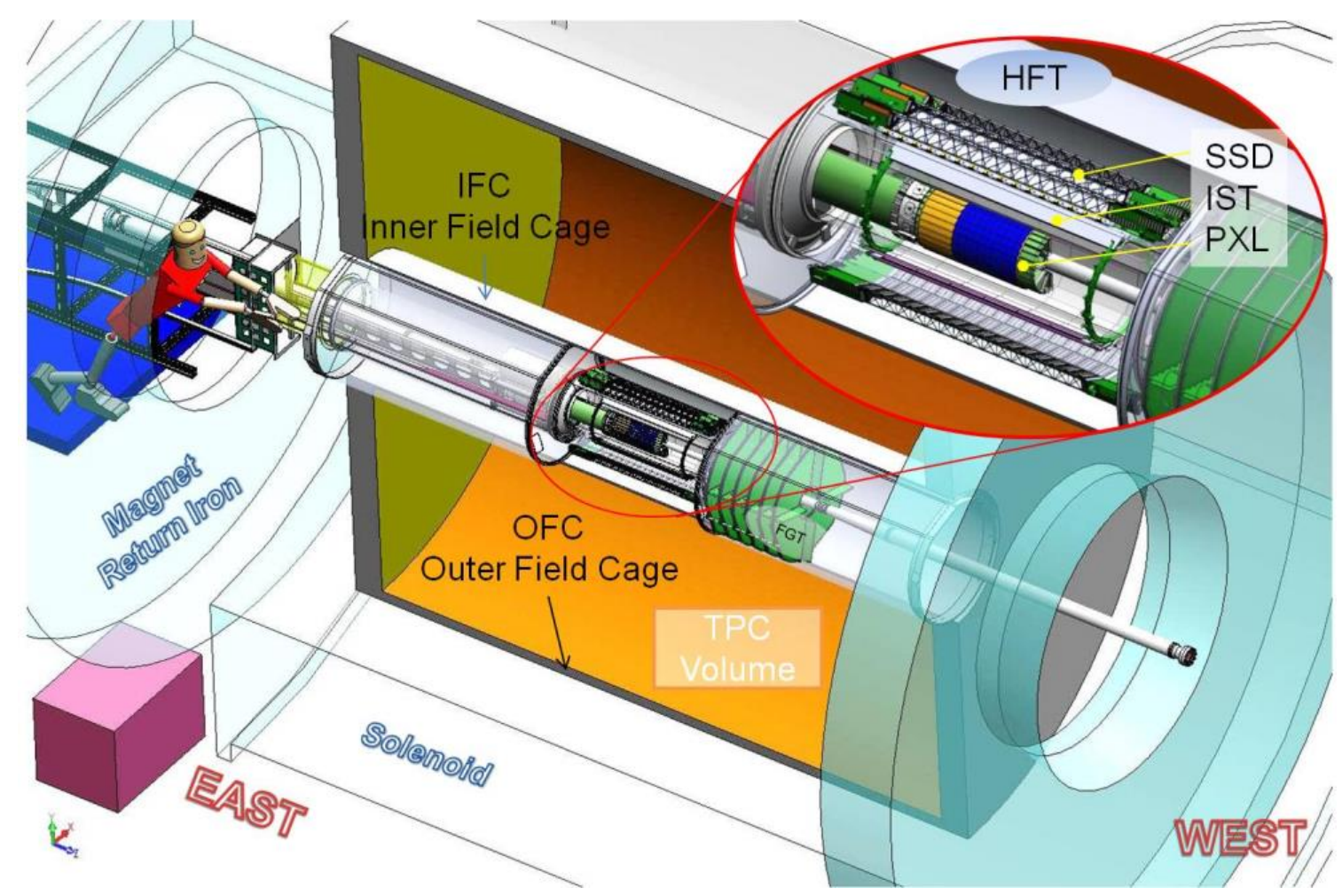

Figure 1 Structure of the STAR HFT upgrade.

\subsection{PXL detector overview}

The CAD structure design of the structure of the HFT upgrade is shown in Figure 1 and the PXL detector is shown as a mixture of photographs and a CAD drawing in Figure 2. 


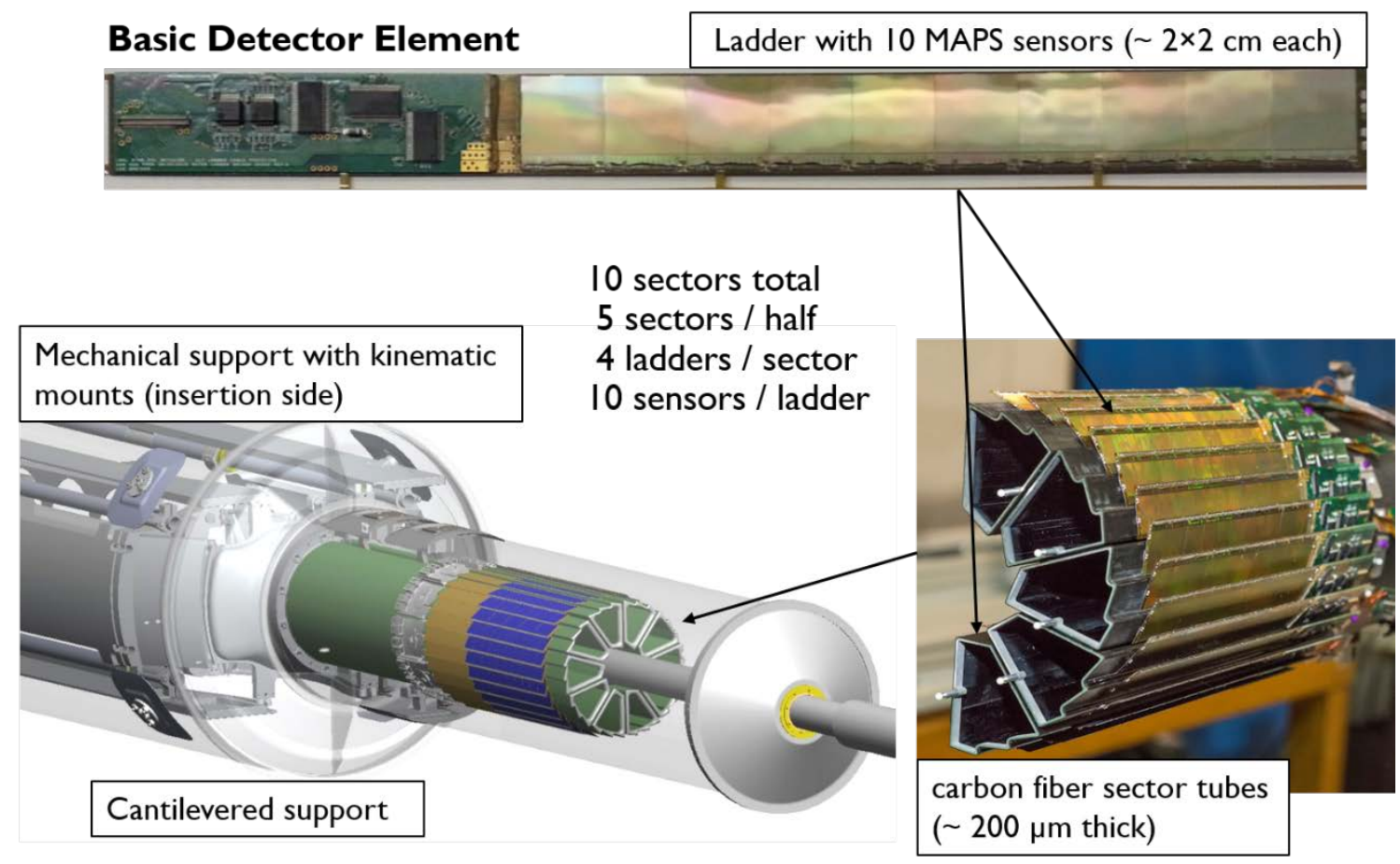

Figure 2 The basic structure of the STAR PXL detector.

The basic element of the PXL detector is a ladder composed of ten $\sim 2 \times 2 \mathrm{~cm}^{2}$ MAPS sensors. The ladders are assembled onto sectors which are composed of three outer ladders and one inner ladder mounted onto a trapezoidal carbon fiber tube. The overall PXL detector is composed of two half shell structures, each with five sectors. The detector is supported and inserted from one side and closes over the beam pipe during insertion locking into kinematic mounts located on the surrounding carbon fiber support tube.

The characteristics of the PXL detector include $356 \mathrm{M}$ pixels on $\sim 0.16 \mathrm{~m}^{2}$ of silicon surface, integration time of $185.6 \mu$ s (to keep the pileup manageable), a DCA pointing resolution (separated into the geometrical and Multiple Coulomb Scattering components) of $10 \oplus 24 \mathrm{GeV} /$ $\mathrm{p} \times \mathrm{C} \mu \mathrm{m}$ for a particle with momentum $\mathrm{p}$, and a radiation length of $0.39 \% \mathrm{X} / \mathrm{X}_{0}$ per layer. In order to meet the stringent radiation length requirements the sensors are thinned down to $50 \mu \mathrm{m}$ and an aluminium conductor based readout flex cable is used on the inner ladders. The $170 \mathrm{~mW} / \mathrm{cm}^{2}$ of heat produced by the sensors is removed using a forced air cooling system that was designed in concert with the mechanics to induce a very low level of vibration due to airflow of $5 \mu \mathrm{m}$ RMS [6]. In addition, the sensors must be able to withstand the modest radiation load imposed by the STAR environment. This load is estimated between 20 to $90 \mathrm{kRad}$ per year and between $2 \times 10^{11}$ to $1 \times 10^{12} 1 \mathrm{MeV} \mathrm{n}_{\mathrm{eq}}{ }^{2} / \mathrm{cm}^{2}$ per year. These characteristics meet the requirements needed to carry out the physics program.

\subsection{Sensors}

The PXL detector achieves several of its crucial characteristics, including high spatial resolution, substantial radiation hardness, and low material budget, by employing sensors

\footnotetext{
${ }^{2}$ Equivalent neutron flux for $1 \mathrm{MeV}$ energy
} 
developed in the Monolithic Active Pixel Sensors (MAPS) technology. The detector is based on custom designed sensors developed by IPHC in Strasbourg, France [3].The sensors used in the PXL detector are designed as $928 \times 960$ pixel arrays of $20.7 \mu \mathrm{m}$ square pixels and have been fabricated in the $0.35 \mu \mathrm{m}$ Austria Micro Systems CMOS process. These sensors include on-chip correlated double sampling, column level discriminators and a zero suppression system that provides hit pixel addresses. These addresses are read out by two Low-Voltage Differential Signal (LVDS) outputs per sensor with a clock speed of $160 \mathrm{MHz}$. The characteristics of this sensor architecture, including performance with a newly available high resistivity silicon process, are described in [3][4][5]. The measured characteristics of the sensors include an efficiency greater than $99 \%$ with an accidental noise hit rate of less than $10^{-5}$ even after exposure to an ionizing radiation dose of $150 \mathrm{kRad}$ and non-ionizing radiation dose of $3 \times 10^{12} 1 \mathrm{MeV} \mathrm{neq} / \mathrm{cm}^{2}$ [5].

The PXL detector Readout (RDO) system is FPGA based and highly parallel, following the mechanical segmentation of the detector with one readout board and one intermediate signal buffering and latch-up protected powering board servicing each detector sector [7].

\section{Project timeline and physics runs}

The HFT project was proposed as a three year physics program starting with an engineering run in 2013 and following with physics running taking Au-Au data in the STAR runs in 2014 and 2016 and proton data in the 2015 run. The initial research and development of this program started as a collaboration with IPHC in Strasbourg in the investigation of the suitability of MAPS sensors for a low mass high resolution vertex detector in 2003. As the sensor development proceeded and the sensor designs improved to the needed level of performance, a proposal was submitted to our funding agency, the US Department of Energy (DOE), to establish the physics case in mid-2006. After the required set of Conceptual and Technical Design reports and project reviews the HFT became a DOE funded construction project in mid-2011. The final physics data taking run was completed in 2016, after which the PXL detector and the rest of the HFT was removed from the STAR detector and put into storage at BNL. The time taken from the physics and detector conception to the initial engineering run of a prototype detector was 10 years. The full physics data set was taken over three consecutive years of physics running and the project was completed on schedule. For a detector of this complexity and using new technology, it required significant effort and planning to install a calibrated detector that could produce usable physics data in the first run.

\section{PXL operational experience}

The PXL detector is the first MAPS based vertex detector at a collider accelerator. As the first generation detector in this technology there were no previous examples to use as a guide for assessing what operational issues may be encountered. The following are the primary set of observations from the operation of the PXL detector over the experimental running periods for the design and operational aspects that worked well. The operational challenges that were encountered will be reported in the "Lessons Learned" section.

- Mechanics and cooling - The detector halves maintained the surveyed pixel positions 
after insertion, during operational heating and in the cooling airflow of $10 \mathrm{~m} / \mathrm{s}$ [6]. The rapid insertion and removal mechanism using kinematic mounts allowed the removal and replacement of a complete operational second detector copy within 24 hours. The air cooling arrangement worked very well. The typical variation in sensor temperature over the runs was within 1-2 degrees $\mathrm{C}$.

- Readout and sensor operation - The current monitoring and Latch-Up (LU) protection circuitry worked well after initial parameter tuning and limited the LU damage to a small number of sensors in a run. The automated Single Event Upset (SEU) sensor reset and reconfiguration scheme on a 15 minute cycle worked well and the SEU rate did not affect the data quality. The PXL RDO system worked well allowing trigger rates up to the STAR TPC maximum $(\sim 1 \mathrm{kHz})$

- Overall - Many aspects of the PXL detector design worked very well from the start. Operational difficulties were encountered but they were overcome and the PXL performance met the requirements to do the physics for which it was designed. The HFT system DCA pointing resolution for $750 \mathrm{MeV} / \mathrm{c}$ kaons was $46 \mu \mathrm{m}$ exceeding the $50 \mu \mathrm{m}$ requirement.
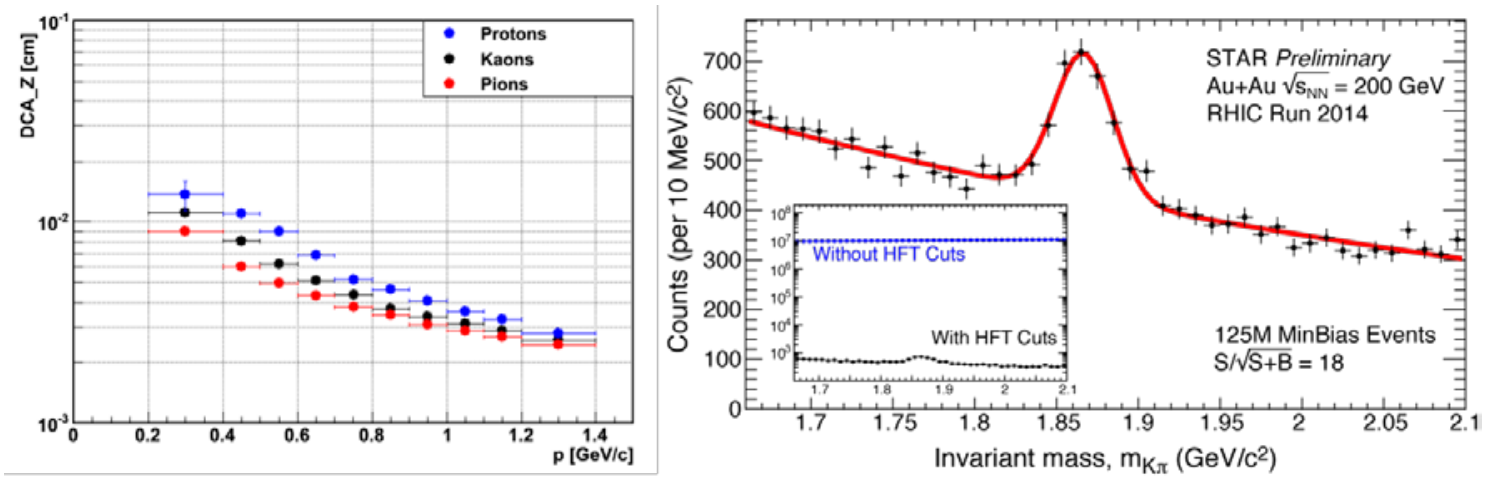

Figure 3 The HFT DCA z pointing resolution is shown on the left (xy resolution is similar). The $D^{0}$ invariant mass reconstruction is shown in figure on the right.

\section{PXL lessons learned}

The prototyping production and running of the PXL detector uncovered a set of lessons learned throughout the detector life highlighting the challenges of realizing a MAPS based vertex detector. The most relevant of these are detailed in the following section.

- It was crucial for us to have planned and scheduled for an engineering run as a check on both the design and the fabrication techniques. During the prototype production of sensor ladders we discovered and fixed problems with the production ladder flex PCB cable solder mask that allowed the thinned sensors to curl down and short out power traces. While installing the detector sectors into the detector half mounting plates, we discovered that there was a mechanical interference between the driver PCBs on the ends of the ladders. We had fully solid modeled the PXL system, nevertheless an error in the sizing of the components on the driver boards was overlooked. This necessitated a redesign of the inner layer structure moving the inner sensor radius from $2.5 \mathrm{~cm}$ to $2.8 \mathrm{~cm}$. In addition, the granularity of our parameter monitoring system was limited to the PXL half-detector and did not allow for an accurate investigation of unexplained current increases. After the 
engineering run, in preparation for the production physics runs, we completely redesigned and re-fabricated the PXL power distribution system to provide monitoring of current and voltage, and to allow for the remote setting of overcurrent thresholds (LU protection) on a ladder basis.

- Some sensor malfunctions were observed after exposure to Au-Au collisions in the startup of the 2014 run. This was accompanied by an increase in ladder current draw on the digital power supply for ladders that showed these sensor malfunctions. These symptom were later attributed to LU induced damage to the sensors. The sensor malfunctions took the form of one or many of the following characteristics: dead sensors, dead rows or columns, sensor half dead, no response from digital control section, and a lack of sensor response to clock or reset. The main focus of the PXL group during the first few weeks was to assess the damage and to try to minimize or stop it from happening. After the first 2 weeks of the run, the rate of damage was dramatically reduced by adjusting the LU current threshold (value at which the power is automatically removed from the ladder) to a point as close to the operating ladder current as possible. After assessing the problem and lowering the LU current threshold from $300 \mathrm{~mA}$ above operating current ( 900mA for an undamaged ladder) to $80 \mathrm{~mA}$ above ladder operating current, we reduced the damage rate from $\sim 1$ sensor/day to $\sim 1$ sensor/month. This and other operational methods such as the previously mentioned 15 minute global reset maintained the same low damage rate of a few sensors per run over the rest of the operational lifetime of the detector. The damage rates may be seen in Figure 4. This low rate of sensor damage was acceptable and limited the damage to the inner layer to approximately $5 \%$ of the inner sensors per running period.

\begin{tabular}{|c|c|c|c|c|c|}
\hline \multirow[t]{2}{*}{ Run } & \multicolumn{2}{|c|}{$\begin{array}{l}\text { Good sensors } \\
\text { on Inner Layer }\end{array}$} & \multicolumn{2}{|c|}{$\begin{array}{l}\text { Good sensors on } \\
\text { Outer Layer }\end{array}$} & \multirow[t]{2}{*}{ Comment } \\
\hline & installation & end of run & installation & end of run & \\
\hline 2014 & $100 \%$ & $82 \%$ & $100 \%$ & $95 \%$ & LU damage, most of it in the first 15 days of operations \\
\hline 2015 & $99 \%$ & $94 \%$ & $98 \%$ & $96 \%(93 \%)^{*}$ & $*=$ Lost control of an outer ladder ( 10 good sensors off $)$ \\
\hline 2016 & $100 \%$ & $95 \%(87 \%)^{+}$ & $99 \%$ & $98 \%$ & ${ }^{+}=$Current instablilty on inner ladder ( 8 good sensors off $)$ \\
\hline
\end{tabular}

Good sensor $=$ sensor with $>95 \%$ active channels and uniform efficiency

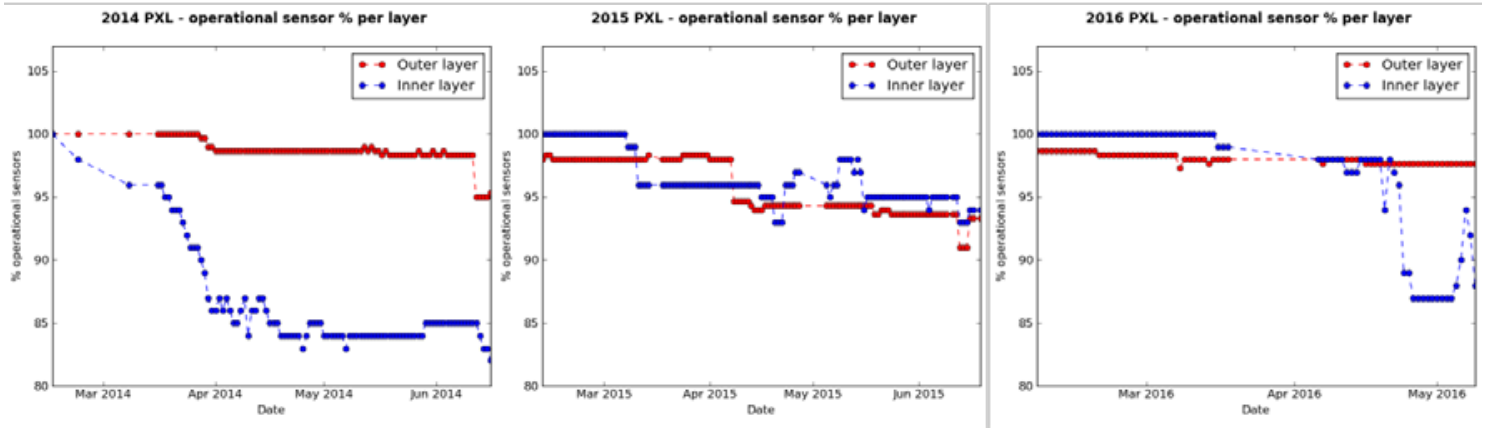

Figure 4 The damage experienced by the PXL detector. Note the initial damage period of 2 weeks in the 2014 run period. After operational methods were put into place to limit PU induced damage, the sensor damage experienced is reduced to a very low rate. 
- After the initial damage, at the end of the 2014 run, the primary PXL detector was removed and the sensors were examined for damage. The damaged sensors showed hot spots when viewed by thermal imaging in the digital section. These hot spots were clustered at particular locations and appeared to be associated with a particular type of buffer structure used in the digital section. Examples of the damaged sensors were taken to the Brookhaven National Laboratory Instrumentation Division for plasma etching and examination of the damaged areas with a Scanning Electron Microscope (SEM). The results can be seen in Figure 5. Sensors at both full thickness of $700 \mu \mathrm{m}$ and thinned to $50 \mu \mathrm{m}$ were taken to the 88” cyclotron at the Lawrence Berkeley National Laboratory for exposure to heavy ion and $55 \mathrm{MeV} / \mathrm{c}$ protons.

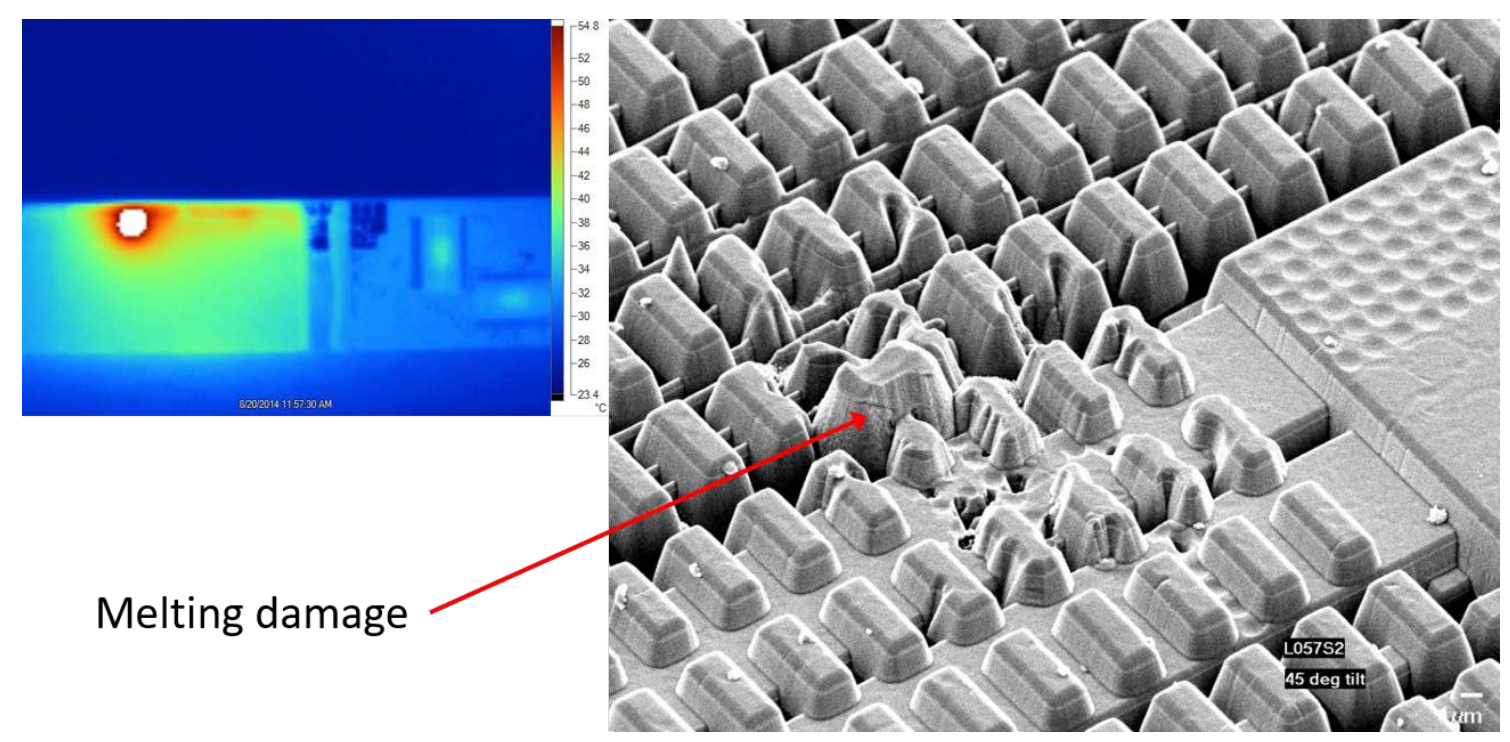

Figure 5 Damage seen on the PXL sensors. A thermal image of a damage location is shown on the left. A SEM photograph of the region showing thermal melting damage is show on the right. The damage is exposed by plasma etching of the sensor surface.

While it was possible to reproduce the damage seen on the thinned $50 \mu \mathrm{m}$ sensors with very large exposures of protons, it was not possible to damage the full thickness sensors despite many attempts and very high LU threshold current limits.

It has been demonstrated by our running experience that there is very low LU damage cross section in a particular silicon structure used in the sensor design. This cross section is too low to reliably find in initial testing and is distinct from LUs in other location on the sensor that do not cause damage. We conjecture that the damage seen is enabled by the fact that the sensors are thinned and thus have less thermal mass and lower thermal conductance to remove heat from a LU point.

- The PXL detector also experienced firmware and software issues during the brief three year running period. A software event decoder bug reduced efficiency for the 2014 Run data. This was discovered and fixed, and the data needed to be reprocessed to recover full efficiency. In addition, at the beginning of the 2015 Run a new set of RDO firmware introduced a subtle bug that caused a partial PXL data loss and reduced the track 
matching efficiency by $\sim 60 \%$ for a significant part of the running period. The bug was discovered and fixed by the 2016 Run.

\section{Conclusions}

The three year physics running of the STAR PXL detector and subsequent physics data analysis indicate that the MAPS technology is suitable for vertex detectors in the RHIC environment. While operational issues were discovered, the problems were overcome and the overall experience using MAPS for the STAR PXL detector was very positive with the physics goals of the program having been achieved. The 2013 Engineering Run was crucial for identifying preproduction problems and allowed us to optimize the designs for the production detectors. As expected for a detector subject to beam-induced damage, the PXL construction phase continued throughout the entire detector life for yearly refurbishment and optimization. The PXL experience demonstrates the difficulty of tuning readout firmware and reconstruction software in the limited time available in a short duration physics program.

\section{Acknowledgements}

We gratefully acknowledge Marc Winter and the PICSEL group at IPHC for the development of the PXL detector sensors. This work was supported by the Director, Office of Science, Office of Nuclear Science of the U.S. Department of Energy under Contract No. DE-AC02$05 \mathrm{CH} 11231$.

\section{References}

[1] J. Adams et al., "Experimental and theoretical challenges in the search for the quark-gluon plasma: The STAR Collaboration's critical assessment of the evidence from RHIC collisions”, Nucl. Phys. A 757 (2005), 102-183.

[2] D.Beavis et al., "The STAR heavy flavor tracker technical design report" https://drupal.star.bnl.gov/STAR/starnotes/public/sn0600 (2011).

[3] M.Winter, "Achievements and perspectives of CMOS pixel sensors for charged particle tracking", Nucl. Instrum. Meth. A 623 (2010), 192-194, doi:10.1016/j.nima.2010.02.192.

[4] C. Hu-Guo et al., "First reticule size MAPS with digital output and integrated zero suppression for the EUDET-JRA1 beam telescope”, Nucl. Instrum. Meth. A 623 (2010), 480-482, doi:10.1016/j.nima.2010.03.043

[5] A. Dorokhov et al., "High-resistivity CMOS pixel sensors and their application to the STAR PIXEL detector”, Nucl. Instrum. Meth. A 650 (2011), 174-177, doi: 10.1016/j.nima.2010.12.112

[6] H. H. Wieman et al., "HFT PXL Vertex Detector Mechanics”, Forum on Tracking Detector Mechanics 2014, https://indico.cern.ch/event/287285/contribution/7

[7] L. Greiner, E. Anderssen, H.S. Matis, H.G. Ritter, J. Schambach, J. Silber, T. Stezelberger, X. Sun, M. Szelezniak, J. Thomas, F. Videbaek, C. Vu, H. Wieman, "A MAPS based vertex detector for the STAR experiment at RHIC,” Nucl. Instr. And Meth. A, vol. 650, pp. 68-72, Sep. 2011 